\title{
Influência do Excesso de Peso Corporal e da Adiposidade Central na Glicemia e no Perfil Lipídico de Pacientes Portadores de Diabetes Mellitus Tipo 2
}

\section{artigo original}

\author{
ana Carolina J. Vasques \\ PATRÍCIA F. Pereira \\ RITA MARIA GOMIDE \\ MARIA CONCEIÇÃo R. BATISTA \\ MARIA TERESA F.S. CAMPOS \\ LUCIANA F.R. SANT'ANA \\ LINA ENRIQUETA F.P.L. ROSADO \\ SILVIA ELOIZA PRIORE
}

Programa Hiperdia da cidade de Viçosa, MG, Disciplina de

Estágio Supervisionado em

Nutrição Social, Departamento de Nutrição e Saúde,

Universidade Federal de Viçosa - UFV, Viçosa, MG.
Recebido em 12/02/07 Revisado em 22/06/07 Aceito em 01/10/07

\section{RESUMO}

O propósito do presente estudo foi avaliar, em pacientes diabéticos do tipo 2, a influência do excesso de peso corporal avaliado pelo IMC e da adiposidade central aferida pela circunferência da cintura (CC) no perfil lipídico sangüíneo e na glicemia de jejum. Foram avaliados 145 indivíduos atendidos em uma unidade de saúde, com idade $\geq 20$ anos. A freqüência do sexo feminino foi de $61,4 \%$. Nos homens, o excesso de peso correlacionou-se com o HDL $(r=-0,34$, $p<0,05)$, colesterol total $(r=0,35, p<0,01)$ e triglicerídeos $(r=0,49, p<0,0001)$, sendo que nas mulheres houve correlação apenas com os triglicerídeos $(r=$ $0,24, p<0,05)$. A CC correlacionou-se com a glicemia de jejum nas mulheres ( $r$ $=0,3, p<0,01)$, com o colesterol total nos homens $(r=0,33, p<0,05)$ e com os triglicerídeos em ambos os sexos ( $F: r=0,22, p<0,05 ; M: r=0,49, p<0,001$ ). Maiores níveis glicêmicos e de triglicerídeos estiveram presentes nos pacientes com excesso de peso e adiposidade central e menores níveis de HDL estiveram associados ao excesso de peso. Conclui-se que há associação entre inadequado perfil metabólico e excesso de peso e/ou adiposidade central, evidenciando a necessidade de intervenção nutricional e clínica em pacientes diabéticos do tipo 2 de forma a reduzir o risco de complicações crônicas futuras. (Arq Bras Endocrinol Metab 2007;51/9:1516-1521)

Descritores: Diabetes mellitus tipo 2; Obesidade; Índice de massa corporal; Circunferência da cintura

\section{ABSTRACT}

Influence of Body Weight Excess and Central Adiposity in Glycemic and Lipid Profile in Patients with Type 2 Diabetes Mellitus.

The purpose of the present study was to evaluate, in diabetic type 2 patients, the influence of body weight excess assessed by BMI and the central adiposity assessed by the waist circumference in the lipid and glycemic profile. One hundred and forty five individuals assisted in a unit of health, aged $\geq 20$ years old, were appraised. The female frequency was $61.4 \%$. Among men, the weight excess was correlated with HDL $(r=-0.34, p<0.05)$, with the total cholesterol $(r=0.35, p<0.01)$, and with the triglycerides $(r=49, p<0.0001)$, and among women there was correlation just with the triglycerides $(r=0.24, p<0,05)$. Waist circumference was correlated with the fast glycemia women's group $(r=$ $0.3, p<0.01)$, with the total cholesterol among men $(r=0.33, p<0.05)$ and with triglycerides in both genders ( $F: r=0.22, p<0.05$; $M: r=0.49, p<0.001$ ). Highest glycemic and triglycerides levels were present in patients with excess body weight and central adiposity and lower HDL levels were present in patients with body weight excess. In conclusion, there is association between inadequate metabolic profile and the excess of body weight and/or central obesity, evidencing the need for a nutritional and clinical intervention in the diabetic type 2 patients, in order to reduce the risk of future chronic complications. (Arq Bras Endocrinol Metab 2007;51/9:1516-1521)

Keywords: Diabetes mellitus type 2; Obesity; Body mass index; Waist circumference 
$\mathrm{O}$ DIABETES MELLITUS DO TIPO 2 (DM 2 ) representa cerca de 90 a $95 \%$ dos casos de diabetes mellitus diagnosticados. Ele é uma desordem metabólica de etiologia múltipla, caracterizada por hiperglicemia crônica, com distúrbios no metabolismo dos carboidratos, gorduras e proteínas, originários de uma defeituosa secreção e/ou ação da insulina nos tecidos-alvo (1).

Os pacientes portadores de DM 2 apresentam mortalidade três vezes maior do que a população em geral, em grande parte devido ao aumento no risco de mortalidade por doença do aparelho circulatório (2).

O primeiro censo nacional de DM realizado no Brasil, no final da década de oitenta, identificou prevalência nacional de $7,6 \%$ e de tolerância diminuída à glicose (pré-diabetes) de $7,8 \%$ na população de 30 a 69 anos, resultando em uma prevalência de 15,4\% (3). Mais recentemente, estudo regional realizado na cidade de Ribeirão Preto, SP, identificou 12,1\% de DM e $7,7 \%$ de tolerância diminuída à glicose (pré-diabetes), perfazendo prevalência de $19,8 \%$ na faixa etária de 30 a 69 anos e retratando aumento da mesma no país com o passar dos anos (4).

$\mathrm{O}$ excesso de peso (sobrepeso e/ou obesidade) está presente em grande parte dos pacientes com DM 2. Na população diabética, em ambos os sexos, um valor de $I M C \geq 25,0 \mathrm{~kg} / \mathrm{m}^{2}$ resulta em aumento na probabilidade de acometimento por doenças cardiovasculares (5). Um estudo multicêntrico brasileiro realizado por Gomes e cols. (2006), com indivíduos portadores de DM 2, constataram que 75\% da amostra estudada não estavam na faixa de peso ideal, sendo que $33,3 \%$ eram obesos (6).

Vários estudos têm sugerido que a variação anatômica da distribuição da gordura corporal é um indicador mais importante de complicações metabólicas do que a massa corporal total (7-10). A obesidade visceral resulta em inúmeras modificações fisiopatológicas, que podem resultar em diferentes graus de resistência à insulina. Dentre elas, podem ser citadas a menor extração de insulina pelo fígado, o aumento na produção hepática de glicose e a diminuição da captação de glicose pelos tecidos periféricos, como o tecido muscular (11). Além da resistência à insulina, a gordura abdominal está relacionada à hipertensão e alterações desfavoráveis no perfil das lipoproteínas plasmáticas, caracterizando o quadro de síndrome metabólica e levando a um aumento no risco de doença cardiovascular (7-10).

À luz destas questões, o presente estudo teve como objetivo avaliar, em pacientes diabéticos do tipo 2 de ambos os sexos, a influência do excesso de peso corporal e da adiposidade central nos parâmetros bioquímicos de controle da doença.

\section{MATERIAL E MÉTODOS}

Trata-se de um estudo epidemiológico, analítico, de delineamento transversal, no qual foram avaliados indivíduos diabéticos atendidos em uma unidade de saúde específica para o atendimento de indivíduos portadores de diabetes e/ou hipertensão arterial da cidade de Viçosa, MG. Consideraram-se como critérios de inclusão indivíduos com diagnóstico de diabetes mellitus tipo 2 e idade $\geq 20$ anos, totalizando 145 indivíduos.

Para avaliação do estado nutricional, bioquímico e clínico, considerou-se peso, estatura, circunferência da cintura (CC), glicemia de jejum, perfil lipídico (colesterol total e frações e triglicérides), pressão arterial sistólica e diastólica e diagnóstico médico de hipertensão arterial.

A partir das medidas de peso e estatura foi calculado o índice de massa corporal (IMC), $\mathrm{em} \mathrm{kg} / \mathrm{m}^{2}$. Considerouse como ponto de corte para excesso de peso o IMC $\geq 25,0$ $\mathrm{kg} / \mathrm{m}^{2}(12)$, sendo agrupados os indivíduos com sobrepeso e obesidade. Para a CC, adotou-se como risco aumentado para o desenvolvimento de complicações metabólicas associadas à obesidade valores $>80 \mathrm{~cm}$ para mulheres e $>94 \mathrm{~cm}$ para homens (13).

Para o diagnóstico de síndrome metabólica, considerou-se a proposta do National Cholesterol Education Program - Adult Treatment Panel III (NCEP-ATP III), o qual leva em consideração, entre seus critérios, a presença de pelo menos três alterações para o diagnóstico da síndrome. Dentre elas estão o uso de medicamentos anti-hipertensivos ou uma pressão arterial $\geq 130 / 85 \mathrm{mmHg}$; níveis de triglicerídeos $\geq 150$ $\mathrm{mg} / \mathrm{dL} ; \mathrm{HDL}<40 \mathrm{mg} / \mathrm{dL}$ (homens) e $<50 \mathrm{mg} / \mathrm{dL}$ (mulheres); glicemia de jejum $\geq 110 \mathrm{mg} / \mathrm{dL}$ e $\mathrm{CC}>102 \mathrm{~cm}$ (homens) e CC $>88 \mathrm{~cm}$ (mulheres) (14).

$\mathrm{Na}$ estatística descritiva, as variáveis qualitativas foram apresentadas em freqüência absoluta e porcentagem, e as quantitativas foram apresentadas, apesar da homogeneidade ou não das variáveis, em média \pm desvio-padrão e mediana (valor mínimo e máximo). Na estatística inferencial foram utilizados testes bicaudais em todas as comparações. Empregou-se o teste estatístico não paramétrico de Mann-Whitney (U) para verificar as diferenças das variáveis entre os sexos, entre os indivíduos eutróficos e com excesso de peso e entre aqueles com e sem adiposidade central. O teste do Qui-quadrado $\left(\chi^{2}\right)$ foi utilizado para análise de proporções. $\mathrm{O}$ coeficiente de correlação de Spearman foi empregado para avaliar a correlação entre os parâmetros antropométricos e bioquímicos.

O processamento e a análise dos dados foram realizados com utilização dos softwares SigmaStat versão 2.03 e do programa EpiInfo versão 6.04. O nível de significância considerado para todas as comparações foi menor que $5 \%$.

Este trabalho foi analisado e aprovado pelo Comitê de Ética em Pesquisa com Seres Humanos da Universidade Federal de Viçosa, MG.

\section{RESULTADOS}

A maior parte da amostra estudada $(61,4 \%)$ era de pacientes do sexo feminino. Comparando-se entre os 
Tabela 1. Características de indivíduos diabéticos atendidos em uma unidade de saúde específica para o atendimento de portadores de diabetes e/ou hipertensão arterial, de acordo com o sexo, da cidade de Viçosa, MG.

\begin{tabular}{|c|c|c|c|c|}
\hline & \multicolumn{2}{|c|}{ Homens $(n=56)$} & \multicolumn{2}{|c|}{ Mulheres $(n=89)$} \\
\hline & Média \pm DP & Mediana & Média \pm DP & Mediana \\
\hline Idade (anos) & $55,5 \pm 9,1$ & $56(36-78)$ & $61,0 \pm 13,6$ & $64(25-82)^{\star *}$ \\
\hline $\mathrm{IMC}\left(\mathrm{kg} / \mathrm{m}^{2}\right)$ & $26,2 \pm 4,2$ & $26(19,5-42,1)$ & $28,7 \pm 4,9$ & $28,1(19,5-48,6)^{\star *}$ \\
\hline $\mathrm{CC}(\mathrm{cm})$ & $93,7 \pm 9,7$ & $94,5(73,5-116)$ & $95,3 \pm 11,5$ & $94(69-129)$ \\
\hline Glicemia de jejum (mg/dL) & $119,4 \pm 58,7$ & $106,7(48-346)$ & $131,9 \pm 54,7$ & $122,5(40-330)$ \\
\hline $\mathrm{HDL}(\mathrm{mg} / \mathrm{dL})$ & $42,6 \pm 12,9$ & $41(21-90)$ & $46,8 \pm 10,2$ & $45(26-86,9)^{\star \star}$ \\
\hline Colesterol total (mg/dL) & $183,3 \pm 41,5$ & $177,9(114-291)$ & $195,1 \pm 34,4$ & $194(119-290)^{*}$ \\
\hline Triglicerídeos (mg/dL) & $131,7 \pm 81,1$ & $108(38-393)$ & $152,9 \pm 79,5$ & $139(44-450)^{*}$ \\
\hline
\end{tabular}

Teste Mann-Whitney

${ }^{*} \mathrm{p}<0,05 ;{ }^{* *} \mathrm{p}<0,01 ;{ }^{* *} \mathrm{p}<0,001$. Dados apresentados em média \pm desvio-padrão (mínimo-máximo). IMC = Índice de Massa Corporal, $C C=$ circunferência da cintura, Excesso de peso $=I M C \geq 25,0 \mathrm{~kg} / \mathrm{m}^{2}$, Adiposidade central $=C C \geq 80 \mathrm{~cm}$ para mulheres e $\mathrm{CC} \geq 94 \mathrm{~cm}$ para homens

Tabela 2. Alterações metabólicas e antropométricas de indivíduos diabéticos atendidos em uma unidade de saúde específica para o atendimento de portadores de diabetes e/ou hipertensão arterial, de acordo com o sexo, da cidade de Viçosa, MG.

\begin{tabular}{lcc}
\hline & Homens $(\boldsymbol{n}=\mathbf{5 6})$ & Mulheres $(\mathbf{n = 8 9 )}$ \\
Dislipidemia & $39(69,6 \%)$ & $60(67,4 \%)$ \\
Hipertensão & $35(62,5 \%)$ & $77(86,5 \%)^{* * *}$ \\
Excesso de peso & $32(57,1 \%)$ & $70(78,6 \%)^{\star *}$ \\
Adiposidade central & $30(53,6 \%)$ & $83(93,2 \%)^{\star * *}$ \\
Síndrome metabólica & $21(37,5 \%)$ & $61(68,5 \%)^{\star * *}$ \\
\hline
\end{tabular}

Teste Qui-Quadrado de proporção

${ }^{*} \mathrm{p}<0,05$; ${ }^{* *} \mathrm{p}<0,01$; ${ }^{* * *} \mathrm{p}<0,001$. Dados apresentados em freqüência absoluta percentual.

sexos, constatou-se que as mulheres apresentaram maiores medianas de idade $(\mathrm{p}<0,01)$, IMC $(\mathrm{p}<0,01)$, colesterol total e triglicerídeos séricos $(\mathrm{p}<0,05)$, excesso de peso $(\mathrm{p}<0,01)$, além de maior freqüência de hipertensão arterial, adiposidade central e síndrome metabólica $(\mathrm{p}<0,001)$. No sexo masculino, a mediana de HDL foi menor $(\mathrm{p}<0,01)$. Não foram encontradas diferenças entre os sexos com relação à freqüência de dislipidemias, entretanto ressalta-se que aproximadamente $70 \%$ dos pacientes de ambos os sexos possuíam pelo menos um tipo de alteração lipídica (tabelas l e 2).

As correlações entre os parâmetros bioquímicos e o excesso de peso foram positivas para os valores de colesterol total e triglicerídeos, e negativa para os níveis de HDL nos pacientes do sexo masculino. Nas mulheres, apenas os níveis de triglicerídeos apresentaram correlação positiva com o excesso de peso (tabela 3).

As correlações entre adiposidade central, medida pela CC, e os parâmetros bioquímicos foram posi-
Tabela 3. Coeficientes de correlação entre parâmetros antropométricos e metabólicos de indivíduos diabéticos atendidos em uma unidade de saúde específica para o atendimento de portadores de diabetes e/ou hipertensão arterial, de acordo com o sexo, da cidade de Viçosa, MG.

\begin{tabular}{lccccc} 
& \multicolumn{3}{c}{ IMC } & \multicolumn{2}{c}{ CC } \\
& Homens & Mulheres & Homens & Mulheres \\
& $\mathbf{r}$ & $\mathbf{r}$ & $\mathbf{r}$ & $\mathbf{r}$ \\
Glicemia de jejum (mg/dL) & 0,22 & 0,17 & 0,20 & $0,30^{* *}$ \\
HDL (mg/dL) & $-0,34^{*}$ & 0,00 & $-0,20$ & $-0,09$ \\
Colesterol total (mg/dL) & $0,35^{* *}$ & 0,03 & $0,33^{*}$ & 0,06 \\
Triglicerídeos (mg/dL) & $0,49^{* * *}$ & $0,24^{*}$ & $0,49^{* *}$ & $0,22^{*}$ \\
\hline
\end{tabular}

Teste de correlação de Spearman

${ }^{*} \mathrm{p}<0,05$; ${ }^{* *} \mathrm{p}<0,01 ;{ }^{* * *} \mathrm{p}<0,001$

tivas para a glicemia de jejum e triglicerídeos nos pacientes do sexo feminino. No sexo masculino, houve correlações positivas entre CC e os níveis de colesterol total e triglicerídeos. Ressalva-se que, mesmo com significância estatística, as correlações encontradas entre os parâmetros antropométricos e metabólicos não foram consideradas fortes (tabela 3 ).

Independentemente do sexo, a comparação entre as medianas dos parâmetros bioquímicos de pacientes com e sem excesso de peso identificou maiores níveis glicêmicos de jejum $(\mathrm{p}<0,05)$ e de triglicerídeos $(\mathrm{p}<0,05)$ naqueles com IMC $\geq 25 \mathrm{~kg} / \mathrm{m}^{2}$. Os homens com excesso de peso apresentaram menores níveis de HDL do que os eutróficos ( $\mathrm{p}<$ $0,05)$ (tabela 4).

A adiposidade abdominal mostrou-se associada aos maiores níveis glicêmicos de jejum em ambos os sexos $(\mathrm{p}<0,05)$. Nas pacientes, os menores níveis de HDL também apresentaram associação com essa localização da gordura $(\mathrm{p}<0,05)$ (tabela 5$)$. 
Tabela 4. Avaliação de parâmetros bioquímicos de indivíduos diabéticos atendidos em uma unidade de saúde específica para o atendimento de portadores de diabetes e/ou hipertensão arterial, de acordo com sexo e a presença de excesso de peso, da cidade de Viçosa, MG.

\begin{tabular}{lcccc}
\hline & \multicolumn{2}{c}{ Homens } & \multicolumn{2}{c}{ Mulheres } \\
& IMC $<25,0$ & IMC $\geq 25$ & IMC $<25$ & IMC $\geq 25, \mathbf{0}$ \\
Glicemia de jejum (mg/dL) & 85,0 & $98,2^{* *}$ & 82,5 & $98,0^{* *}$ \\
HDL (mg/dL) & 43,3 & $37,0^{*}$ & 51,0 & 44,5 \\
Colesterol total (mg/dL) & 174,9 & 179,0 & 203,0 & 193,0 \\
Triglicerídeos (mg/dL) & 95,5 & $133,5^{* *}$ & 108,0 & $158,2^{*}$ \\
\hline
\end{tabular}

Teste Mann-Whitney

${ }^{*} \mathrm{p}<0,05 ;{ }^{* *} \mathrm{p}<0,01$

Tabela 5. Avaliação de parâmetros bioquímicos de indivíduos diabéticos atendidos em uma unidade de saúde específica para o atendimento de portadores de diabetes e/ou hipertensão arterial, de acordo com sexo e adiposidade central, da cidade de Viçosa, MG.

\begin{tabular}{lcccc}
\hline & \multicolumn{2}{c}{ Homens } & \multicolumn{2}{c}{ Mulheres } \\
& $\mathbf{C C} \leq \mathbf{9 4 , 0}$ & $\mathrm{CC}>\mathbf{9 4 , 0}$ & $\mathrm{CC} \leq \mathbf{8 0 , 0}$ & $\mathbf{C C}>\mathbf{8 0 , 0}$ \\
Glicemia de jejum (mg/dL) & 91,2 & $97,0^{*}$ & 89,0 & $97,0^{*}$ \\
HDL (mg/dL) & 41,0 & 40,2 & 52,5 & $44,0^{*}$ \\
Colesterol total (mg/dL) & 181,2 & 176,9 & 170,5 & 197,5 \\
Triglicerídeos (mg/dL) & 97 & 120,5 & 100,5 & 146,0 \\
\hline
\end{tabular}

Teste Mann-Whitney

${ }^{*} \mathrm{p}<0,05$

\section{DISCUSSÃo}

Nas últimas décadas, tem sido observado incremento na prevalência de DM2. No contexto do processo de transição nutricional, esse incremento tem sido atribuído às modificações do estilo de vida, ao aumento das taxas de sobrepeso e obesidade e ao envelhecimento populacional (15). Apesar de o DM2 ser o tipo de diabetes mais comum, seu diagnóstico precoce não é freqüente, uma vez que o estado hiperglicêmico não implica, necessariamente, em sintomas clínicos perceptíveis (1).

$\mathrm{O}$ excesso de peso corporal, assim como a adiposidade visceral em indivíduos diabéticos do tipo 2, tem sido associado a um pior perfil lipídico, a elevados níveis pressóricos e à presença de síndrome metabólica $(1,16)$, o que torna essencial o conhecimento acerca da influência do excesso de peso e sua distribuição no controle do DM2 em pacientes ambulatoriais para um melhor tratamento e controle da doença.

Neste estudo, os pacientes não apresentaram um adequado controle glicêmico, uma vez que médias de glicemia de jejum, em ambos os sexos, encontraram-se acima do ponto de corte de $100 \mathrm{mg} / \mathrm{dL}$ estabelecido para um bom controle do diabetes (1). É importante salientar que a permanência de níveis glicêmicos elevados relaciona-se com sérias complicações crônicas microangiopáticas (retinopatia diabética, nefropatia diabética e neuropatia sensitiva distal) e macroangiopáticas (cardiopatia isquêmica, doença vascular periférica e acidente vascular cerebral) de alta prevalência em pacientes diabéticos ambulatoriais $(1,17)$.

A presença da síndrome metabólica é um achado comum nos diabéticos do tipo 2 (18), o que pôde ser verificado pela elevada freqüência de síndrome metabólica encontrada no presente estudo $(35,7 \%$ nos homens e $68,5 \%$ nas mulheres).

Segundo uma meta-análise de estudos de coorte prospectivos, indivíduos diabéticos apresentam risco de doença coronariana significativamente maior do que os saudáveis (19). Entretanto, dentre os diabéticos, ainda existem diferenças no risco cardiovascular entre os sexos. A presença de maior risco de mortalidade por doença cardiovascular em mulheres diabéticas do que em homens diabéticos relatado em outros trabalhos (19-21) também foi identificada na amostra estudada. No que se refere aos parâmetros avaliados, as mulheres apresentaram pior controle metabólico do diabetes, além de maiores freqüências de hipertensão 
arterial, excesso de peso com adiposidade central e síndrome metabólica, todos esses importantes fatores de risco para o desenvolvimento de complicações cardiovasculares.

O excesso de peso corporal exerce grande influência na sensibilidade à insulina e está diretamente associado ao desenvolvimento do DM2. É consenso que a distribuição anatômica do tecido adiposo está relacionada a alterações indesejáveis no perfil lipídico, dentre outros vários efeitos deletérios da obesidade $(22,23)$.

Diferenças regionais na lipólise induzida pelas catecolaminas e nos efeitos antilipolíticos da insulina têm sido demonstrados. Em ambos os sexos, independentemente do grau de obesidade, os adipócitos localizados nas regiões femurais e glúteas exibem resposta lipolítica mais branda do que os adipócitos viscerais localizados na região abdominal (24).

Um dos mecanismos plausíveis mais propostos para explicar a relação entre adiposidade visceral e resistência à insulina considera a grande sensibilidade à estimulação dos receptores $\beta$-adrenérgicos em comparação com os receptores $\alpha$-adrenérgicos localizados no tecido adiposo visceral (25). Por esse mecanismo, ocorreria maior liberação de ácidos graxos não esterificados na corrente sanguínea a partir dos adipócitos viscerais, maior captação de ácidos graxos pelo fígado, aumento na produção de VLDL, substrato para a gliconeogênese e, como resultado final, um estado de hipertrigliceridemia e hiperglicemia $(25,26)$.

As correlações encontradas entre o excesso de peso e a adiposidade visceral com o perfil lipídico e glicêmico também foram identificadas em um estudo realizado com homens diabéticos, o qual identificou controle glicêmico prejudicado, menores níveis de HDL além de uma freqüência maior de doença vascular periférica, doença cardiovascular isquêmica e hipertensão naqueles com adiposidade central, demonstrando a associação entre o excesso de gordura abdominal, com ou sem a manifestação de obesidade, com um pior controle metabólico do diabetes (27).

Em conclusão, o presente estudo identificou a associação entre inadequados perfis lipídicos e níveis glicêmicos de jejum mais elevados com a presença de excesso de peso e/ou adiposidade central, evidenciando a necessidade de intervenção imediata em pacientes diabéticos do tipo 2 com excesso de peso ou adiposidade central para melhor controle metabólico da doença e menor risco de complicações crônicas futuras. Ressalta-se que especial atenção deva ser destinada aos pacientes diabéticos do sexo feminino de Viçosa, MG, uma vez que as mesmas apresentaram maior freqüência de fatores de risco além de pior con- trole metabólico da doença, estando em maior risco cardiovascular que os homens. Assim, medidas conservadoras como educação nutricional, acompanhamento dietético e incentivo à prática de atividade física devem ser constantemente implementadas de forma a auxiliar na perda e manutenção de peso corporal em pacientes ambulatoriais portadores de DM 2.

\section{REFERÊNCIAS}

1. American Diabetic Association. Diagnosis and classification of diabetes mellitus. Diabetes Care 2006;29(suppl. 1):S43-8.

2. Salles GF, Bloch KV, Cardoso CRL. Mortality and predictors of mortality in a cohort of Brazilian type 2 diabetic patients. Diabetes Care 2004;27:1299-305.

3. Malerbi DA, Franco LJ. Multicenter study of the prevalence of diabetes mellitus and impaired glucose tolerance in the urban Brazilian population aged 30-69 yr. The Brazilian Cooperative Group on the Study of Diabetes Prevalence. Diabetes Care 1992;15:1509-16.

4. Torquato MT, Montenegro Junior RM, Viana LA, De Souza RA, Lanna CM, Lucas JC, et al. Prevalence of diabetes mellitus and impaired glucose tolerance in the urban population aged 30-69 years in Ribeirão Preto (São Paulo), Brazil. São Paulo Med J 2003;121:224-30.

5. Castro SH, Mato HJ, Gomes MB. Parâmetros antropométricos e síndrome metabólica em diabetes tipo 2. Arq Bras Endocrinol Metab 2006;50:450-5.

6. Gomes MB, Giannella Neto D, De Mendonça E, Tambascia MA, Fonseca RM, Réa RR, et al. Prevalência de sobrepeso e obesidade em pacientes com diabetes mellitus do tipo 2 no Brasil: Estudo multicêntrico nacional. Arq Bras Endocrinol Metab 2006;50:136-44.

7. Guedes DP, Guedes EP. Controle do peso corporal em populações jovens In: Controle corporal: Composição corporal, atividade física e Nutrição. Londrina: Midiograf, 1998.

8. Pereira A, Sichieri R, Marins VM. Razão cintura/quadril como preditor de hipertensão arterial. Cad Saúde Pública 1999;15:333-3.

9. Nieves DJ, Cnop M, Etzlaff B, Walden CE, Brunzell JD, Knopp $\mathrm{H}$, et al The Atherogenic lipoprotein profile associated with obesity and insulin resistence is largely attributable to intraabdominal fat. Diabetes 2003;52:172-9.

10. Giorgino F, Laviola L, Eriksson JW. Regional differences of insulin action in adipose tissue: insights from in vivo and in vitro studies. Acta Physiol Scand 2006;183:13-30.

11. DeNino WF, Tchernof A, Dionne IJ, Toth MJ, Ades PA, Sites CK, et al. Contribution of abdominal adiposity to age-related differences in insulin sensitivity and plasma lipids in healthy nonobese women. Diabetes Care 2001;24:925-32.

12. World Health Organization. Obesity: Preventing and managing the global epidemic. Geneva, 1997.

13. Han TS, van Leer EM, Seidell JC, Lean ME. Waist circumference action levels in the identification of cardiovascular risk factors: prevalence study in a random sample. Br Med J 1995;311:1401-5.

14. Sociedade Brasileira de Diabetes. Atualização brasileira sobre diabetes. Rio de Janeiro: Diographic, 2006.

15. Sartorelli DS, Franco JL. Tendências do diabetes mellitus no Brasil: o papel da transição nutricional. Cad Saúde Pública 2003;19(sup.1):S29-S36.

16. Rodriguez BL, Curb JD, Burchfiel CM, Huang B, Sharp DS, Lu $\mathrm{GY}$, et al. Impaired glucose tolerance, diabetes and cardiovascular disease risk factor profiles in the elderly. The Honolulu Heart Program. Diabetes Care 1996;19:587-90.

17. Scheffel RS, Bortolanza D, Weber CS, Costa LA, Canani LH, Santos KG, et al. Prevalência de complicações micro e macrovasculares e de seus fatores de risco em pacientes com diabetes melito do tipo 2 em atendimento ambulatorial. Rev Assoc Med Bras 2004;50:263-7. 
18. Siekavizza JL, Perez FG. A doença cardiovascular no diabético. Curso Latino-Americano sobre Diabetes e Síndrome Metabólica para Clínicos. 2003. cap. 3. p. 22.

19. Huxley R, Barzi F, Woodward M. Excess risk of fatal coronary heart disease associated with diabetes in men and women: meta-analysis of 37 prospective cohort studies. BMJ 2006;332:73-8.

20. Wei M, Gaskill SP, Haffner SM, Stern MP. Effects of diabetes and level of glycemia on all-cause and cardiovascular mortality. The San Antonio Heart Study. Diabetes Care 1998;21:1167-72.

21. Corrêa FHS, Taboada GF, Júnior CRMA, Faria AM, Clemente ELS, Fuks AG, et al. Influência da gordura corporal no controle clínico e metabólico de pacientes com diabetes mellitus tipo 2. Arq Bras Endocrinol Metab 2003;47:62-8.

22. Ohlson LO, Larsson B, Svardsudd K, Welin L, Eriksson H, Wilhelmsen $L$, et al. The influence of body fat distribution on the incidence of diabetes mellitus: 13.5 years of follow-up of the participants in the study of men born in 1913. Diabetes 1985;34:1055-8.

23. Montague CT, O'Rahilly S. The perils of portliness: causes and consequences of visceral adiposity. Diabetes 2000; 49:883-8.
24. Mlinar B, Marc J, Janex A, Pfeifer M, Molecular mechanisms of insulin resistance and associated diseases. Clin Chem Acta 2007;375:20-35.

25. Frayn KN. Visceral fat and insulin resistance - causative or correlative? Br J Nutr 2000;83(suppl. 1):S71-7.

26. Rosenbaum P, Ferreira SRG. An update on cardiovascular risk of metabolic syndrome. Arq Bras Endocrinol Metab 2003;47:220-7

27. Van Gaal L, Rillaerts E, Creten W, De Leeuw I. Relationship of body fat distribution pattern to atherogenic risk factors in NIDDM: Preliminary results. Diabetes Care 1988;11:103-6.

Endereço para correspondência:

Ana Carolina Junqueira Vasques Rua Gomes Barbosa 343, apto. 201 36570-000 Viçosa, MG 